\title{
CARACTERIZACIÓN HISTOPATOLÓGICA DE GASTRITIS ASOCIADA A LA PRESENCIA DE Helicobacter spp EN ESTÓMAGOS DE CABALLOS
}

\section{CHARACTERI ZATI ON HISTOPATOLOGI CAL OF GASTRITIS ASSOCI ATED TO Helicobacter SPP PRESENCE I N STOMACHES OF HORSES}

\author{
José Cardona Á, ${ }^{1 *}$ M.SC, Enrique Paredes H, ${ }^{2}$ Ph.D, Heriberto Fernández, ${ }^{3}$ Ph.D. \\ IUniversidad de Córdoba, Facultad de Medicina Veterinaria y Zootecnia, Departamento \\ de Ciencias Pecuarias, Área de Clínica Medico-Quirúrgica de Grandes Animales. Montería, \\ Colombia. ${ }^{2}$ Universidad Austral de Chile. Facultad de Ciencias Veterinarias. Instituto de \\ Patología Animal. Valdivia, Chile. ${ }^{3}$ Universidad Austral de Chile. Facultad de Medicina. \\ Instituto de Microbiología Clínica. Valdivia, Chile. * Correspondencia: \\ cardonalvarez@hotmail.com
}

Recibido: Agosto 18 de 2008; Aceptado: Junio 18 de 2009.

\section{RESUMEN}

Objetivo. Caracterizar el tipo de lesión histopatológica asociada a la presencia de Helicobacter spp. en úlceras gástricas de caballos. Materiales y métodos. Se utilizaron 25 muestras de estómagos de caballos con úlceras positivas en 2 o mas pruebas diagnósticas a bacterias curvoespiraladas Helicobacter spp. Se analizó en forma descriptiva el tipo de lesión histopatológica y el grado de la úlcera gástrica, mediante la tinción de hematoxilina-eosina. A todas las muestras, se le determinó su grado y el tipo de gastritis asociada, clasificándolas en tres grupos de categorías según el grado de intensidad de las úlceras, establecida por el Consejo del Síndrome de Úlceras Gástricas en Equinos (CEGUS) y la clasificación de la gastritis asociada se realizó según la clasificación de Sydney modificada, Houston 1995. Se consideraron las variables del total de animales, zona anatómica (segmento de la mucosa gástrica) y edad. Resultados. El 30\% de las úlceras fueron grado 1 y 2 , de las cuales $15 \%$ presentaron gastritis crónica, $12.5 \%$ gastritis crónica activa y un $2.5 \%$ gastritis eosinofílica crónica. El $57.5 \%$ de las muestras presentaron úlceras grado 3 y 4 , de las cuales $25 \%$ presentó gastritis crónica activa, $17.5 \%$ gastritis eosinofílica crónica y el $15 \%$ gastritis crónica. Sólo el $12.5 \%$ de las úlceras fueron grado 5 y 6 , de las cuales $7.5 \%$ presentó gastritis crónica activa, $2.5 \%$ gastritis crónica y el $2.5 \%$ restante presentó gastritis eosinofílica crónica. Conclusiones. Las úlceras gástricas grado 3 y 4 fueron las de mayor presentación, microscópicamente la gastritis crónica activa y la gastritis crónica fueron las más observadas en las muestras positivas a Helicobater spp en dos o más pruebas diagnósticas.

Palabras clave: Gastritis, Helicobacter spp, úlceras, caballos. 


\section{ABSTRACT}

Objective. To Characterize the type of histopatological lesion associated to Helicobacter spp presence in gastric ulcers of horses. Materials and methods. It was inclueded 25 horses of stomaches with sings of positive ulcers in 2 or more diagnostic test to curvedspiral bacteria Helicobacter spp. A Description of histopathological type of lesion, degree of gastric ulcer was done by hematoxylin-eosin staining. In all samples of gastric ulcers, it determines the degree and type of gastritis associated, and classified into three categories by degree of severity of ulcers, established by the council's Syndrome, Gastric Ulcers in Equines (CEGUS) and the classification of the associated gastritis was performed according to modified Sydney classification, Houston 1995. Variables considered were the total of animals, anatomical area (gastric segment of the mucous one) and age. Results. $30 \%$ of the ulcers were grade 1 and 2, $15 \%$ of them presented as chronic gastritis, $12.5 \%$ chronic active gastritis and a $2.5 \%$ chronic eosinofílic gastritis. $57.5 \%$ of the signs showed ulcers grade 3 and $4,25 \%$ of them presented as chronic active gastritis, $17.5 \%$ chronic eosinofílic gastritis and the $15 \%$ chronic gastritis. Only $12.5 \%$ of the ulcers were grade 5 and 6 , of which $7.5 \%$ presented chronic active gastritis, chronic gastritis $2.5 \%$ and $2.5 \%$ remaining chronic eosinophilic gastritis. Conclusions. The gastric ulcers grade 3 and 4 were the ones belonging to bigger presentation; microscopically the chronic active gastritis and the chronic gastritis were the more observed in the positive signs to Helicobater spp in two or more diagnostic tests.

Key words: Gastritis, Helicobacter spp, gastric, horses.

\section{NTRODUCCI ÓN}

Está demostrado que la infección por bacterias del genero Helicobacter spp, desempeña un papel importante en el desarrollo de diversas enfermedades digestivas tanto en humanos como en animales (1). Actualmente se acepta que este microorganismo está relacionado con la presentación de gastritis crónica activa. Este tipo de gastritis, es un proceso patológico multicausal, por lo que se presta para confusión desde el punto de vista clínico e histopatologico (2). La primera respuesta de la mucosa gástrica frente a la infección por Helicobacter spp, parece ser una gastritis aguda con infiltración epitelial de neutrófilos sin otra celularidad inflamatoria acompañante (3). Los factores iniciadores de gastritis crónica (Helicobacter spp, acidez, etc) inducen una respuesta neutrofílica y la participación de los polimorfonucleares neutrófilos (PMN) permeando el epitelio glandular y constituyen la denominada "actividad inflamatoria" de las gastritis activas o en actividad, también llamadas gastritis crónicas agudizadas (4). Las patologías gástricas de los caballos disminuyen considerablemente su rendimiento, principalmente aquellos que se encuentran en competencia, exposiciones o trabajo (4). El objetivo del presente estudio fue determinar el tipo de gastritis asociada a la presencia de bacterias curvo espiraladas tipo Helicobacter spp. en úlceras gástricas de caballos.

\section{MATERI ALES Y MÉTODOS}

Sitio de estudio y recolección de muestras. El presente estudio en su parte práctica se realizó en el I nstituto de Patología Animal de la Facultad de Ciencias Clínicas de la Universidad Austral de Chile. Las muestras fueron obtenidas en la planta faenadora de carnes de Chol-Chol, ubicada a $28 \mathrm{~km}$ al noroeste de Temuco (IX Región, Chile). Se utilizaron 25 muestras de estómagos con úlceras positivas a bacterias curvoespiraladas tipo Helicobacter spp, 
identificadas como positivas en 2 o mas pruebas diagnósticas como test de ureasa, Gram directo y Whartin Starry. Identificandose las bacterias como ureasa positiva al cambio de color del reactivo de naranja-amarillo a rosa fucsia y por su morfología característica (curvadas o espiraladas) mediante tinción de Gram y por su afinidad por tinciones de Whartin Starry.

Procesamiento de las muestras. Cada una de las muestras positivas a Helicobacter spp fue sometida a tinción de Hematoxilinaeosina. Las muestras para examen histopatológico fueron procesadas en el Instituto de Patología Animal de la Facultad de Ciencias Veterinarias de la Universidad Austral de Chile. Las muestras fueron laminadas y procesadas automaticamente en un procesador de tejidos (Shandon-Elliott. Bench $\AA$, SCE 0400, Darmstadt, Alemania) a fin de ser deshidratadas e impregnadas en parafina. Finalmente fueron incluidas en parafina sólida, cortadas mediante micrótomo a 5-6 micras de grosor y teñidas con Hematoxilina-eosina, con el fin de analizar el tipo de lesión histopatológica asociada a la presencia de bacterias tipo Helicobacter $\operatorname{spp}(5,6)$.

Caracterización histopatológica de las lesiones. A todas las muestras se les determinó su grado y tipo de gastritis asociada, clasificándolas en tres grupos de categorías según el grado de intensidad de las úlceras, establecida por el Concejo del Síndrome de Úlceras Gástricas en Equinos (7) y la clasificación de la gastritis asociada se realizó según la clasificación de Sydney modificada, Houston 1995 (8, 9).

\section{RESULTADOS}

\section{Graduación y caracterización histopatológica de las úlceras positivas a Helicobacter spp. En el presente estudio todas las muestras fueron tomadas desde úlceras gástricas, determinándose su grado y el tipo de gastritis asociada.}

La figura 1, muestra la graduación de las úlceras y su clasificación histopatológica, en

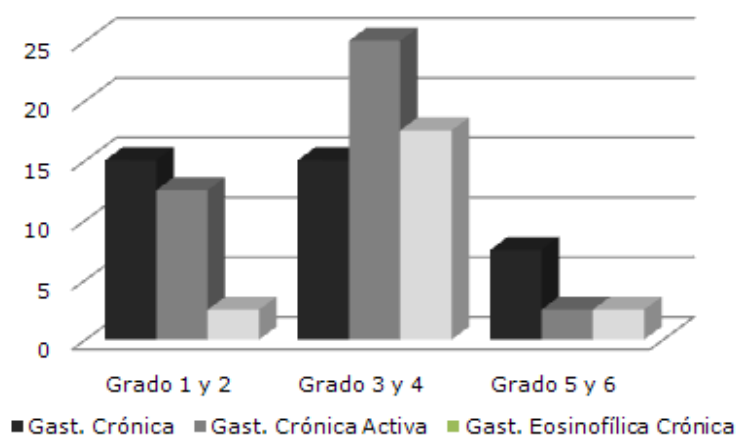

Figura 1. Porcentaje de muestras, según el grado de la úlcera gástrica, en 25 caballos positivos a Helicobacter spp.

los 25 caballos positivos a Helicobacter spp. En el primer grupo se reunieron las muestras con úlceras grado 1 y 2 , encontrándose un $30 \%$, de las cuales $15 \%$ presentaron gastritis crónica, $12.5 \%$ gastritis crónica activa y un $2.5 \%$ gastritis eosinofílica crónica. El segundo grupo, reunió las muestras con úlceras grado 3 y 4 (Figura 2), encontrándose más de la mitad de la población en estudio con $57.5 \%$, en la cual el $25 \%$ presentó gastritis crónica activa (Figura 3), 17.5\% gastritis eosinofílica crónica (Figura 4) y el 15\% gastritis crónica. En el tercer grupo se reunieron las muestras con grado 5 y 6 , siendo el grupo con menor número de muestras con $12.5 \%$, de las cuales $7.5 \%$ presentó gastritis crónica activa, $2.5 \%$ gastritis crónica y el $2.5 \%$ restante presentó gastritis eosinofílica crónica.

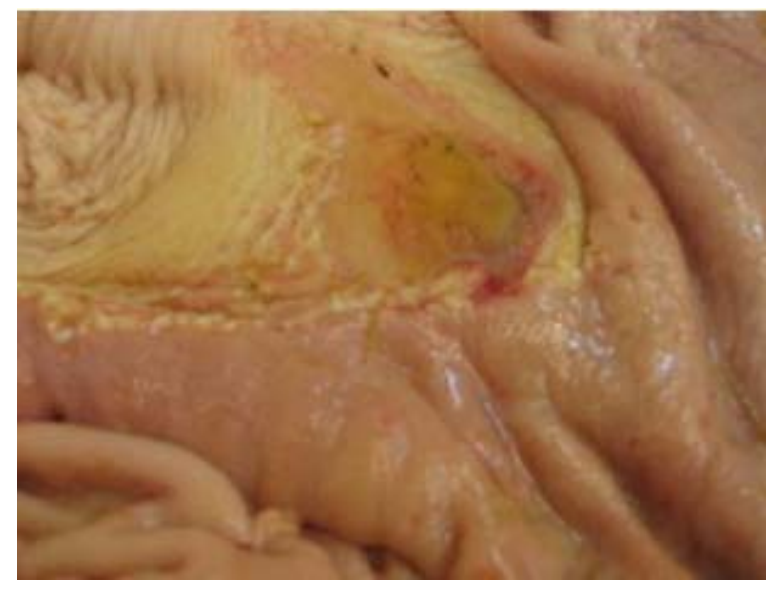

Figura 2. Úlcera gástrica grado 4 con gastritis crónica activa asociada, nótese la solución de continuidad en la mucosa gástrica. 


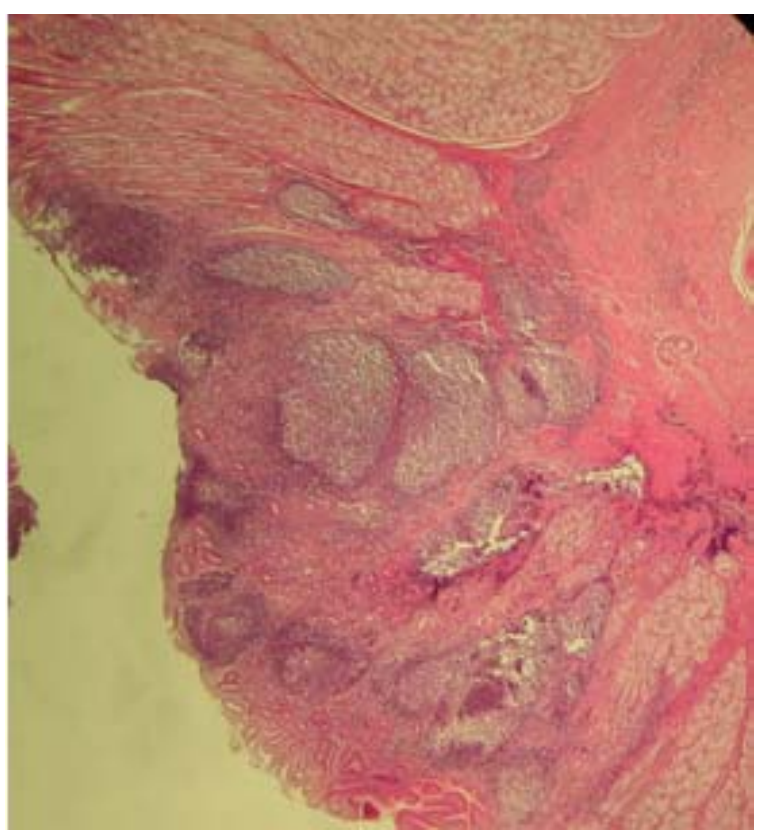

Figura 3. Ulcera gástrica grado 4 con gastritis crónica activa asociada, nótese la severa reacción inflamatoria con abundante infiltrado inflamatorio de carácter neutrofilico. $4 \mathrm{X}$.

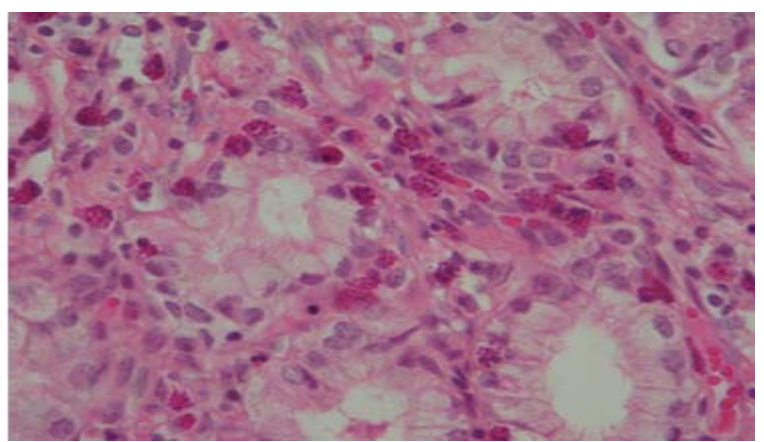

Figura 4. Gastritis eosinofílica crónica asociada, nótese la severa reacción inflamatoria con abundante infiltrado inflamatorio de carácter eosinofilico. 10X

Frecuencia de distribución de bacterias tipo Helicobacter spp. en úlceras gástricas, según edad. La figura 5 , muestra los porcentajes de presentación de bacterias tipo Helicobacter spp. según edad, con respecto a las diferentes zonas de la mucosa gástrica donde fueron tomadas las muestras y resultaron positivos. Se observa mayor predominio de positividad para Helicobacter spp. en el segundo grupo etáreo ( 6 a 10 años) para ambas zonas (fúndica y antral), siendo mayor en la región fúndica. En los animales del primer grupo etáreo ( 1 a 5 años), resultaron positivos a la bacteria en test de ureasa y Whartin Starry en un $2.5 \%$ en la región fúndica y $2.5 \%$ en la región antral. En los animales pertenecientes al segundo grupo etáreo ( 6 a 10 años) se observó una frecuencia de presentación de $27.5 \%$ en la región fúndica y $15 \%$ en la región antral, siendo éste el grupo con mayor frecuencia de presentación de animales positivos a las dos pruebas. En el tercer grupo de animales ( 11 a 15 años), se observó una frecuencia de presentación del $7.5 \%$ en la región fúndica y $2.5 \%$ en la región antral. En el último grupo de animales (16 a 20 años), se pudo observar un $2.5 \%$ de presentación en la región fúndica y $2.5 \%$ en la región antral.

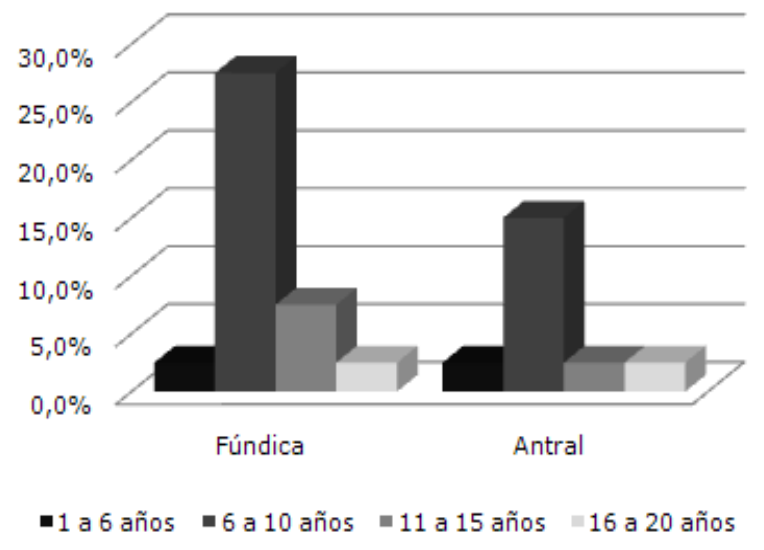

Figura 5. Frecuencia de presentación de bacterias úlceras gástricas en 25 caballos positivos a bacterias tipo Helicobacter spp. en dos o tres pruebas según edad y zona de la mucosa gástrica afectada.

\section{SCUSI ÓN}

Graduación y caracterización histopatológica de las úlceras positivas a bacterias tipo Helicobacter spp. a dos o tres pruebas en 25 caballos. El segundo grupo (úlceras grado 3 y 4) fue el de mayor presentación de muestras positivas a bacterias tipo Helicobacter spp, seguido del primer grupo (úlceras grado 1 y 2), mientras que el tercer grupo (úlceras grado 5 y 6 ), presentó un escaso porcentaje de muestras positivas.

En cuanto al tipo de gastritis asociada en las muestras positivas a bacterias tipo 
Helicobacter spp. se encontraron muestras con gastritis crónica, gastritis crónica activa y gastritis eosinofílica crónica, siendo la gastritis crónica activa la de mayor presentación, seguida de gastritis crónica, principalmente en las úlceras con grado 3 y 4 , seguidas de los grados 1 y 2 , mientras que en las úlceras grado 5 y 6 , sólo hubo un pequeño número de muestras con gastritis crónica activa y mínimo porcentaje de muestras que presentaron gastritis eosinofílica crónica.

Estos datos coinciden con los reportados por diversos autores (10-12), quienes indican que el proceso inflamatorio de la mucosa infectada con bacterias tipo Helicobacter spp., cursa con gastritis crónica y gastritis crónica activa. De igual forma Contreras et al (13), encontraron 7 caballos con úlceras gástricas y 5 con gastritis en animales positivos a Helicobacter spp en una población de 20 pacientes equinos. Asimismo Araya et al (4), encontraron que un alto porcentaje de los casos presentó una actividad inflamatoria significativa con polimorfonucleares (PMN), categorizándolas como gastritis crónica activa (Gastritis crónica agudizada).

Por otra parte Moyaert et al (14), reportaron un porcentaje considerable de pacientes neonatos equinos positivos a Helicobacter equorum con manifestaciones clínicas digestivas, aunque no se estableció el tipo de gastritis asociada, así como Valenzuela et al (15), demostraron la presencia de organismos espiroidales gástricos desde biopsias provenientes del fundus gástrico, obtenidas por gastroscopía en potros clínicamente sanos, pero no establecieron histopatológicamente la presencia o no de lesión gástrica.

Sin embargo, es prescindible recordar la capacidad de la bacteria de estimular la producción de ureasa, la cual participa en la colonización de la mucosa gástrica y en la sobrevivencia del microorganismo en el pH ácido del estómago. Más aún, la ureasa por sí misma y el amonio como producto final del desdoblamiento, tienen una función importante en la inflamación induciendo la respuesta inmune, ya que el amonio actúa como factor quiomiotáctico que activa a los monocitos y leucocitos polimorfonucleares para liberar citocinas, ocasionando una respuesta inflamatoria localizada con daño del tejido del epitelio gástrico $(16,17)$.

Por lo expresado en los diagnósticos histológicos más frecuentes (gastritis crónica y gastritis crónica activa) y su asociación con el Helicobacter spp., se puede establecer que existe una asociación entre el diagnóstico histológico encontrado y la infección por esta bacteria (11-14). Los resultados de este estudio indican que un alto número de los pacientes con gastritis crónica y gastritis crónica activa están infectados por bacterias con morfología compatible con Helicobacter spp., lo que sugiere, que la infección por esta bacteria sería la causa más importante de la gastritis crónica y gastritis crónica activa en equinos adultos. No obstante, los casos de pacientes con gastritis crónica, gastritis crónica activa tienen una prevalencia de la infección por Helicobacter spp netamente más alta que la que presentan los pacientes con histología normal (11).

En los casos donde se presentó gastritis eosinofílica crónica, se observaron cifras de eosinófilos bastantes superiores al $5 \%$ en el tejido, cifra a partir de la cual se considera patológico. Dentro de los posibles diagnósticos diferenciales de gastritis eosinofílica crónica y que cursen con manifestaciones digestivas, se encuentran las infecciones del tubo digestivo por parásitos del género Trichostrongylus y Gasterophylus (18). Esto coincide con lo observado en este estudio, ya que los parásitos antes mencionados, fueron observados macroscópicamente el día de la toma de muestras y en el microscopio se observaron formas parasitarias en la tinción de hematoxilina-eosina.

\section{Agradecimientos}

Al Instituto de Patología Animal, al Instituto de Ciencias Clínicas de la Facultad de Ciencias Veterinarias de la Facultad de Medicina de la Universidad Austral de Chile, al Instituto de Microbiología Clínica de la Facultad de Medicina de la Universidad Austral de Chile. 


\section{REFERENCI AS}

1. Gómez L, Orozco S, Salas S. Helicobacteriosis canina y felina. Vet Mex 2006; 37: 97 - 116.

2. González-Carbajal M, Sevilla L, Grá L. Alteraciones histológicas de la mucosa gástrica y prevalencia del Helicobacter pylori en pacientes dispépticos. Rev Panam Infectol 2005; 7: 8-15.

3. Chillihua K, Palomino R, Aguilar E. Aislamiento de Helicobacter pylori a partir de biopsias gástricas de pacientes con gastritis en el hospital regional del Cusco, Perú. Situa 2005; 13: 15- 19.

4. Araya J, Villaseca M, Roa I, Roa J. Helicobacter pylori y gastritis crónica: relación entre infección y actividad inflamatoria en población de alto riesgo de cáncer gástrico. Rev Méd Chile 2000; 128: 259 - 265.

5. Luna L. Manual of histologic methods of the armed forces institut of pathology. $3^{\text {th }}$ ed. New York, USA: McGraw-Hill; 1968.

6. López-Brea M, Alarcón T, Baquero M, Domingo D, Royo G. Diagnóstico de la infección por Helicobacter pylori. En: Cercenado E, Cantón R. Procedimientos en Microbiología Clínica. Recomendaciones de la Sociedad Española de Enfermedades Infecciosas y Microbiología Clínica. 2004; 17: 1- 10.

7. Bertone J. Equine gastric ulcer syndrome. In: $10^{\circ}$ congresso nazionale multisala sive. Perugia, Italia. 2004.

8. Dixon M, Genta R, Yardley J, Correa P. Classification and grading of gastritis. The Updated Sydney System. Am J Surg Pathol 1996; 20: 1161-1181.

9. Genta R. Recognizing Atrophy: Another step toward a classification of gastritis. Am J Surg Pathol 1996; 20: 523 - 530.
10. Blaser M. Helicobacter pylori and the pathogenesis of gastroduodenal inflammation. J Infect Dis 1990; 161: $626-633$.

11. Moyaert $H$, Decostere A, Pasmans F, Baele M, Ceelen L, Smits K, Ducatelle $R$, Haesebrouck F. Acute in vivo interactions of Helicobacter equorum with its equine host. Equine Vet $\mathrm{J} 2007$; 39(4): 370-372.

12. Piñol F, Paniagua M. Mediadores bacterianos de la inflamación en la gastritis crónica por Helicobacter pylori. Rev Cub Med 1999; 38: 276 - 283.

13. Contreras M, Morales A, García-Amado MA, De Vera M, Bermúdez V, Gueneau P. Detection of Helicobacter-like DNA in the gastric mucosa of Thoroughbred horses. Lett Appl Microbiol 2007; 45(5): 553-557.

14. Moyaert H, Haesebrouck F, Dewulf J, Ducatelle R, Pasmans F. Helicobacter equorum is highly prevalent in foals. Vet Microbiol 2009; 133(1-2): 190-192.

15. Valenzuela O, Luzio A, Muñóz L, Urrutia P, García A. Detección de organismos espiroidales gástricos en estómago de potrillos clínicamente sanos. Avances en Ciencias Veterinarias 2004; 19: 40 - 45.

16. Berg D, Hoffman P, Appelmelk B, Kusters J. The Helicobacter pylori Genome Sequence: Genetic Factors for Long Life in the Gastric Mucosa. Trends Microbiol 1997; 5: 468 - 473.

17. Marshall D, Dundon W, Beesley S, Smyth C. Helicobacter pylori a Conundrum of Genetic Diversity. Microbiol 1998; 144: 2925 - 2939.

18. Madroñal N, Martin E, Gil E. Gastritis eosinofílica: a propósito de un caso. MEDIFAM 2003; 13: 111 - 115. 